\title{
Homogenous Segmentation based Edge Detection Techniques for Proficient Identification of the Cotton Leaf Spot Diseases
}

\author{
P. Revathi \\ Research Scholar, Karpagam \\ University,Coimbatore-21.TamilNadu, India
}

\author{
M. Hemalatha \\ Head, Dept of Software System, Karpagam \\ University,Coimbatore-21.TamilNadu, India
}

\begin{abstract}
In this work we express technological strategies using mobile captured symptoms of cotton leaf spot images and classify the diseases using neural network. The system has been trained to achieve intelligent farming for rural area farmers, including early recognition of diseases in grows, selective fungicide application,etc..This research work proposes an automatic image preprocessing techniques. At first, the captured images are processed for improvement. Other edge detectors presented in earlier works can detect edges on different size objects. In this Research work, a homogeneity operator can take the difference of the center pixel and a pixel that is two or three pixels away. The major objective of this Research work is to use Homogeneity-based edge detector segmentation, which takes the result of any edge detector and divides it by the average value of the area. This work has been implemented in the real time software and produces best results.
\end{abstract}

\section{Keyword}

Homogenous Edge detection, Image Segmentation, Neural Network, Cotton leaves f spot.

\section{INTRODUCTION}

Image segmentation is an important aspect of digital image processing. Image segmentation may be defined as a process of assigning pixels to homogenous and disjoint regions which form a partition of the image that share certain visual characteristics. Homogeneity operator can take the difference of the center pixel and a pixel that is two or three pixels away. The main target of this work is using Homogeneity-based edge detector segmentation, which takes the result of any edge detector and divides it by the average value of the area. This division removes the effect of uneven lighting in the image. The average value of an area is available by convolving the area with a mask containing all ones and dividing by the size of the area.

\section{LITERATURE REVIEW\& RELATED STUDIES}

Presented studies carried out in [5] propose a Biogeography Based optimization approach for image segmentation i.e. partitioning an image into multiple segments. And used for training with support vector machines (SVSM) for cotton leaf disease segmentation [6] another work image segmentation of cucumber leaf images is proposed. First, the color space model is analyzed.[7] a kind of color feature is applied to obtain the feature map, which combines RGB model and HSI model. [8] we determine the RGB threshold value, through counting the characteristic parameter of stochastic picture element in the different image spot.[9] Segmentation is the most important part to detect paddy disease early and accurately, we presented an application of image processing technique and a new method Gaussian Mean (GM) for segmenting paddy disease.[10] Image Segmentation to identify regions in the image that were likely to qualify as diseased region, Image Feature Extraction and Selection to extract and select important image features and Image Classification to classify the image into different herbs diseases classes.[11]Image segmentation techniques to identify infected part of the plants. [12] The lesion areas with anthracnose and frog-eye spots on a leaf of tobacco seedlings are segmented by contrast stretching transformation with an adjustable parameter and morphological operations. [13] In this work novel approach combines low-cost NIR filters used on a standard camera with neural networks to achieve a significantly higher accuracy as compared to classic threshold techniques. [14] First the maize leaf disease pictures of different varieties were taken in the fields; the images were deniosed and segmented. [15] Edge detection refers to the process of identifying and locating sharp discontinuities in an image. The main of this paper aim is to survey the theory of edge detection for image segmentation using soft computing approach based on the Fuzzy logic, Genetic Algorithm and Neural Network. [16] In this presented work was Edgeflow-driven Geometric Snake or EDGS.The partial differential equation (PDE) resulting from this integration of image Edgeflow and region term is implemented using a level set approach.

\section{EDGE DETECTION PROCESS IN IMAGE SEGMENTATION}

"Segmentation" correlated [5] to the process of separating a digital image into multiple segments such as a set of pixels, also known as super pixels (Chad and Hayit, 2002). The major goal of segmentation is to simplify and/or change the representation of an image into meaningful image that is more proper and easier to explore. Segmentation is essentially a collection of methods that allowing spatially partitioning close parts of the image as objects. Edge identification techniques convert images to edge images benefiting from the changes of gray tones in the images. Edges are the sign of lack of continuity, and ending. As a result of this conversion, edge image is obtained without 
encountering any changes in the physical qualities of the main image [1] [2]. Objects consist of numerous parts of different color levels. In an image with different gray levels, despite an obvious change in the gray levels of the object, the shape of the image can be distinguished. An Edge in an image is a significant local change in the image intensity, usually associated with a discontinuity in either the image intensity or the first derivative of the image intensity. Discontinuities in the image potency can be either Step edge, where the image intensity abruptly changes from one value on one side of the discontinuity to a different value on the opposite side, or Line Edges, where the image intensity abruptly changes value but then returns to the starting value within some short distance [3]. However, Step and Line edges are rare in real images. Because of low frequency components or the smoothing introduced by most sensing devices, sharp discontinuities rarely exist in real signals. Step edges become Ramp Edges and Line Edges become Roof edges, where intensity changes are not instantaneous but occur over a finite distance [4]. In this work, Homogeneity-based edge detector segmentation is used to take the result of any edge detector and divides it by the average value of the area. This division removes the effect of uneven lighting in the image. The average value of an area is available by convolving the area with a mask containing all ones and dividing by the size of the area. Ultimately getting the cotton leaves spot diseases affected part is very bright and boundary way to identify each cotton leaves spot diseases easy to identify that diseases part of the edge segmentation process.

\section{TYPES OF COTTON LEAF SPOT DISEASES}

The diseases on the cotton leaves are classified as

- Fusarium wilt

- Verticillium wilt

- Root rot

- Boll rot

- Grey mildew

- Leaf blight

- Bacterial blight

- Leaf curl

\section{5. HOMOGENEOUS SEGMENTATION BASED EDGE DETECTION (HSBED) METHOD}

The other edge detectors presented so far can detect edges on different size objects. The homogeneity operator can take the difference of the center pixel and a pixel that is two or three pixels away. The difference edge detector can take the difference of opposite pixels in $5 \times 5$ or $7 \times 7$ areas instead of a $3 \times 3$ area.

The Homogeneity-based edge detector takes the result of any edge detector and divides it by the average value of the area. This division removes the effect of uneven lighting in the image. The average value of an area is available by convolving the area with a mask containing all ones and dividing by the size of the area.
Proposed Algorithm (HSBED)

Input: A Sample Image from Segmentation

Output: Detected Edges

Step 1: Accept the input image

Step 2: Apply mask Gx, Gy to the input image.

Step3:Apply Sobel and canny edge detection with homogenes operator algorithm and the gradient (Homogeneous).

Step 4: Masks manipulation of Gx, Gy separately on the input image.

Step 5: Results combined to find the absolute magnitude of the gradient.

$$
|\mathrm{G}|=\sqrt{ }(\mathrm{Gx} 2+\mathrm{Gy} 2)
$$

Step 6: the absolute magnitude is the output edges.

\section{EXPERIMENTAL RESULTS}

Take the Image a leaf as an input image and convert into a grayscale image, and by using Canny and Sobel Edge detection techniques and with the use of homogenous operator the images are combined and through this we obtain the image clarity for the edges, and we get centered pixel of opposite neighboring one, two, three pixels of white lightning. This lightning identified is denoted as the diseases affected is represented in the edges of leaf boundary. The dissimilarity edge detector can take the dissimilarity of opposite pixels in instead of a $3 \times 3$ area.

The Homogeneity-based edge detector takes the result of any edge detector and divides it by the average range of the part. This division removes the effect of not level lighting in the image. The average range of an area is available by convolving the part with a mask containing all ones and separating by the size of the area.

\section{FUSARIUM WILT}

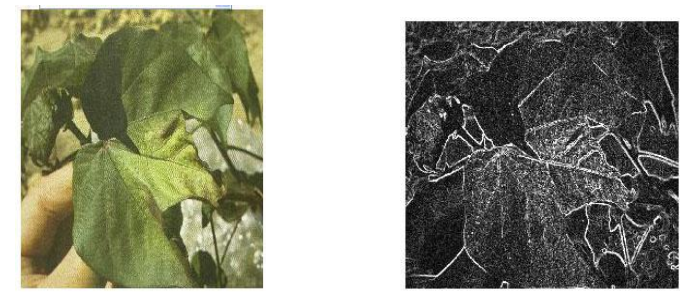

Figure (a1) Input Image Figure (a2) Invert using Homogeneous edge detection

\section{VERTICILLIUM WILT}
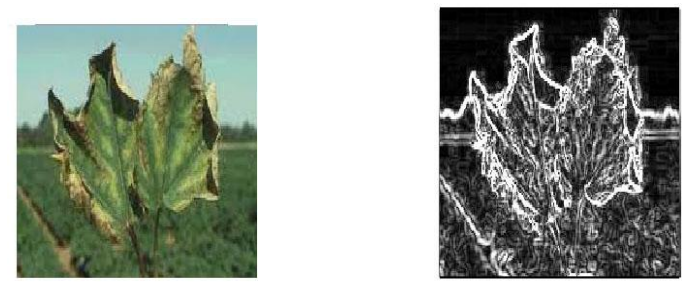

Figure (b1) Input Image Figure (b2) Invert using Homogeneous edge detection. 


\section{LEAF BLIGHT}
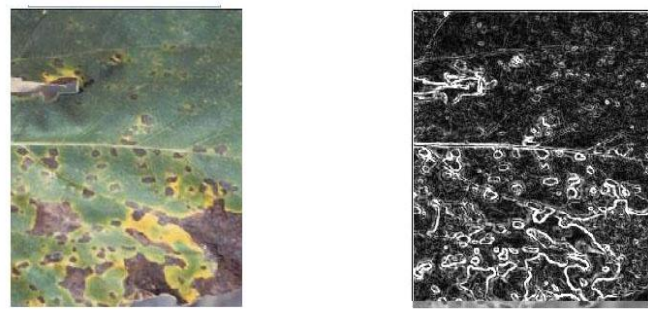

Figure (c1) Input Image

Figure (c2) Invert using Homogeneous edge detection.

This work is analyzed with eight types of disease in cotton leaves. The above figures show the example for edge detected diseases. Results are for three types of cotton leaves. The figure a1.Fusarium Wilt and Figure b1.Verticillium Wilt. Figures c1.Leaf Blight is shown after the disease are recognized. In the figures a2, b2\& c2 homogenous edge detection techniques are shown in fair light part is diseases find out that part.

\section{ACKNOWLEDGMENTS}

I would like to express my special thanks of gratitude to Dr.S.Nakkeeran SevugaPerumal, Associate Professor (Pathology) Department of Plant Pathology of Tamil Nadu Agriculture University,and my guide Dr.M.Hemalatha,Head of Dept Software System in Karpagam University had given valuable ideas in my Research work. I thank and I will be gratitude them for overall supports.

\section{CONCLUSION}

Segmentation is a collection of methods allowing interpreting spatially close parts of the image as objects. Edge detection techniques transform images into edge images benefiting from the changes of gray tones in the images. Edges are the sign of lack of continuity, and ending. In this work achieving the goal of Homogeneity-based edge detector takes the result of any edge detector and divides it by the average value of the area. This division removes the effect of uneven lighting in the image. The average value of an area is available by convolving the area with a mask containing all ones and dividing by the size of the area. And train and test using a neural network classifier. Then classification the diseases and achieving very excellent accuracy, the software is very rapid and time intense, lessen the cost, automatically identify the diseases and pest recommendation to farmers through a mobile phone. The proposed work is very helpful to agriculture sectors and farmers immediately taking a decision and reduce their burden,life safety and Recommedation to the pest control it.This work has been implemented and producing fruitful results.

\section{REFERENCES}

[1] Ibrahiem M. M. El Emary, "On the Application of Artificial Neural Networks in Analyzing and Classifying the Human Chromosomes", Journal of Computer Science, Vol.2 (1), 2006, pp.72-75.

[2] N. Senthilkumaran and R. Rajesh, "A Study on Edge Detection Methods for Image Segmentation", Proceedings Of the International Conference on Mathematics and Computer Science (ICMCS-2009), 2009, Vol.I, pp.255-259.
[3] M. Abdulghafour,"Image segmentation using Fuzzy logic and genetic algorithms", Journal of WSCG, vol. 11,No.1, 2003.

[4] Metin Kaya,"Image Clustering and Compression Using an Annealed Fuzzy Hopfield Neural Network",

[5] International Journal of Signal Processing, 2005, pp.80-88.

[6] Surbhi Gupta, Krishma Bhuchar, Parvinder S.Sandhu, "Implementing Color Image Segmentation Using Biogeography Based Optimization" International Conference on Software and Computer ApplicationsIPCSIT Vol.9 (2011) () (2011) IACSIT Press, Singapore.

[7] Jiazhi Pan,Young He. "Recognition of plants by leaves digital image and neural network"IEEE proceedings on 2008 International Conference on Computer Science and Software Engineering.

[8] Lidi Wang,Tao yang and YouwenTian" Crop Disease Leaf Image Segmentation Method Based on Color Features" Proceedings of the 2007 International Conference on Wavelet Analysis and Pattern Recognition, Beijing, China, 2-4 Nov. 2007.

[9] Wang Jiaofei; Wang Shuangxi; Cui Yanli; Coll. of Eng., Shanxi Agric. Univ. ,Taigu, China, "Research on the color image segmentation of plant disease in the greenhouse" Consumer Electronics, Communications and Networks (CECNet), 2011 International Conference on Issue Date: 16-18 April 2011 On page(s): $2551-2553$.

[10] Md. Zahangir Alom Hyo Jong Lee" Gaussian Mean Based Paddy Disease Segmentation" 2010 the 3rd International Conference on Machine Vision (ICMV 2010), ISBN: 978-1-4244-8889-6 C ICMV 2011.Pg: no: 522-525.

[11] Lili N.A, F. Khalid, N.M. Borhan, "Classification of Herbs Plant Diseases via Hierarchical Dynamic Artificial Neural Network after Image Removal using Kernel Regression Framework" ISSN: 0975-3397 Vol. 3 No. 1 Jan 2011, (IJCSE) PG: No: 15-20.

[12] Phadikar,S.Sil, J.;"Rice disease identification using pattern recognition techniques" Computer and Information Technology, 2008. ICCIT 2008. 11th International Conference on, ISBN: 978-14244-2135-0, Pg: No: 420 - 423.

[13] S.Guru,P.B,Mallikarjuna,S.Manjunatha,"Segmen tation and classification of tobacco seedling diseases" Bangalore Compute Conf of IEEE, 2011, pp.32-32.

[14] Floris De Smedt, Ive Billauws and Toon Goedemé" Neural Networks and Low-Cost Optical Filters for Plant Segmentation" International Journal of Computer Information Systems and Industrial Management Applications. ISSN 2150-7988 Volume 3 (2011) pp. 804-811. 
[15] Chen Li1,Wang Lanying" Research on Application of Probability Neural Network in Maize Leaf Disease Identification"journal of Agriculture Mechanization Research 2011.

[16] N.Senthilkumaran and R. Rajesh," Edge Detection Techniques for Image Segmentation A Survey of Soft Computing Approaches" International Journal of Recent Trends in
Engineering, Vol. 1, No. 2, May 2009, Pg: No: 250-254.

[17] Ying Yang,Xin Gao,'’Image Segmentation Using Edgeflow-Driven Geometric Snake " Proceedings of the 2007 International Conference on Wavelet Analysis and Pattern Recognition, Beijing, China, 2-4 Nov. 2007,Pg:No:505-509. 